\title{
Parameters Identification of Synchronous Machine Based on Particale Swarm Optimization
}

\author{
Bendaoud Elrachid ${ }^{1, *}$, Radjeai Hammoud ${ }^{1, * *}$, and Boutalbi Oussama ${ }^{2, * * *}$ \\ ${ }^{1}$ LAS Laboratory, Electrical Engineering Department, Setif 1 University, Setif, Algeria. \\ ${ }^{2}$ LSI Laboratory, Electronic Department, Setif 1 University, Setif, Algeria.
}

\begin{abstract}
This paper, deals with a meta-heuristic method, the Particle Swarm Optimization (PSO), for operational parameters identification of synchronous machine. The considered method consists of minimizing quadratic criterion that represents the difference between simulated model at standstill frequency response output and those computed from the model to be identified. The obtained results by simulation show that the method based on particle swarm optimization is efficient in terms of convergence speed and gives optimal solution.
\end{abstract}

\section{Introduction}

In the last years, there has been a growing interest to the field of accurate determination of the $d-q$ axis equivalent circuit parameters for the simulation of synchronous machines. Much analytical methods such as Marquardt algorithm [1], Hartley Series [2], Volterra Series [3], maximum likelihood estimation (MLE) [4] have been proposed.

In [5], an Artificial Neural Network (ANN) based modelling technique for the rotor body parameters of synchronous generator is developed and tested. In [6], a Genetic Algorithms (GAs) approach is used to identify the desired parameters. Another method based on Hybrid genetic algorithms is used in [7] to find high-order equivalent circuits of synchronous machines. Although GAs are efficient in finding the global minimum of the search space and the solutions are independent on initial values of the parameters, they consume too much search time which is not proper for online identification reported in the literature[1] . Compared to GA, Particle Swarm Optimization (PSO) approach is easy to implement, converges quickly and it was improved and widely used in parameters identification [8-10].

The current paper presents a model-based parameter identification algorithm for synchronous machine equivalent circuit using particle swarm optimization. The results obtained by simulation showed that the method based on PSO is efficient in not only in terms of convergence speed, but also in terms of finding of the optimal values. The paper is organized as follows: in Section 2, we introduce the mechanism of particle swarm optimization. Section 3 describes the Standstill Frequency Response (SSFR) test. PSO based parameter identification method is presented in section 4. In Section 5, the application of the presented

\footnotetext{
*e-mail: bendaoud.elrachid@gmail.com

**e-mail: hradjeai@yahoo.fr

***e-mail: botalbioussama@gmail.com
}

method is carried out in the MATLAB environment. Section 6 concludes the paper.

\section{Particle swarm optimisation}

The PSO algorithm is originally attributed to Dr. Eberhart and Dr. Kennedy, which is one of the latest efficient evolutionary techniques inspired from the animals social behaviour as fish schooling, bird flocking [11][10]. The PSO is a population-based search process where each particle of the swarm moves randomly along a search space and interacting with each other [12][11]. In the PSO algorithm, each particle remember its best personal solution found and best solution acquired by the whole swarm. By using this information (best personal solution and best solution of the whole swarm) each particle, update its position as given in Eq.1. In each iteration, the velocity is updated using the following equation.

$$
v_{k+1}=w(k) \cdot v_{k}+c_{1} \cdot r_{1}\left(l b p-c p_{k}\right)+c_{2} \cdot r_{2}\left(g p_{k}-c p_{k}\right)
$$

The new velocity is added to the current particle position $c p_{k}$ to generate new particle position $c p_{k+1}$, as shown in Eq.2:

$$
c p_{k+1}=c p_{k}+v_{k+1}
$$

where $w$ is the inertia weight varied between initial value and final value as following:

$$
w=\left(\text { iter }_{\max }-\text { iter }_{\text {cur }}\right)\left(\frac{w_{\text {initial }}-w_{\text {final }}}{\text { iter }_{\max }}\right)+w_{\text {find }}
$$

Where $w_{\text {initial }}$ and $w_{\text {find }}$ represent the initial and final inertia weights at the start of a given run, respectively; iter $_{\text {max }}$ is the maximum number of iterations in an offered run, and iter $_{\text {curent }}$ denotes the current iteration number at the present time step [12]. $c_{1}$ and $c_{2}$ are two positive parameters called acceleration coefficients which represent the 


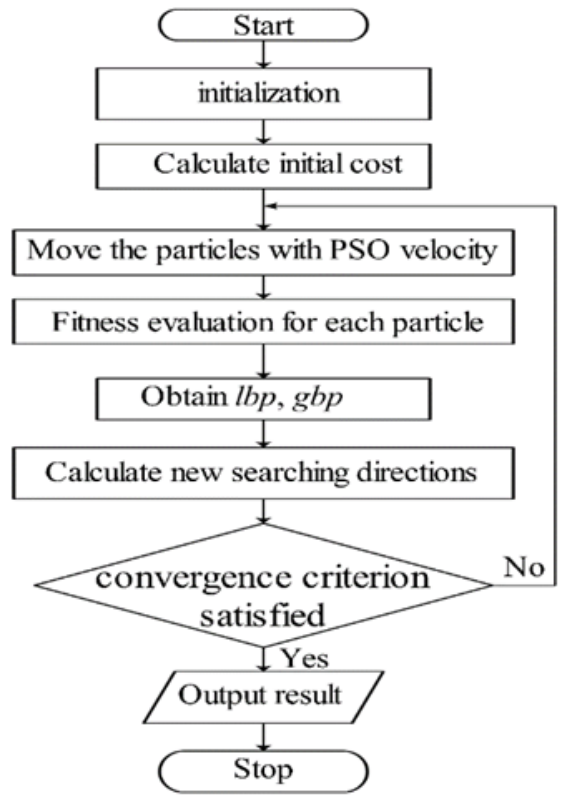

Figure 1. Flowchart of the PSO algorithm.

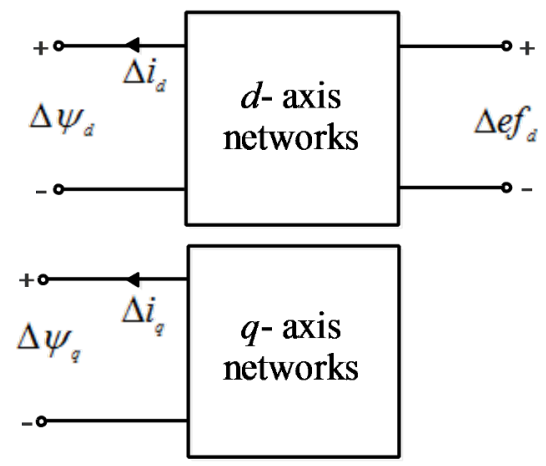

Figure 2. The d- and q-axis networks identifying terminal quantities.

individual and sociality weights coefficients for modelling attractive forces from the local and global best solution, respectively. Usually, $c_{1}=c_{2}$ ranges from 0 to 4 [11-13]. The implementation procedure of the PSO algorithm is illustrated with the flowchart in Fig.1.

\section{Two-axis equivalent circuit of the synchronous machine}

This approach represents an adequate method to identify the electrical characteristics of a synchronous machine which is defined by the operational parameters that relate the armature and the filed terminal quantities. Referring to Fig.2, the relationship between the armature and the field terminal quantities can be expressed in the operational form as [14]:

$$
\begin{aligned}
& \Delta \psi_{d}(s)=G(s) v_{f}(s)-L_{d}(s) i_{d}(s) \\
& \Delta \psi_{q}(s)=-L_{q}(s) i_{q}(s)
\end{aligned}
$$

where, $s$ is the familiar Laplace operator, $G(s)$ is the armature-to-field transfer function, $L_{d}(s)$ is the d-axis op-

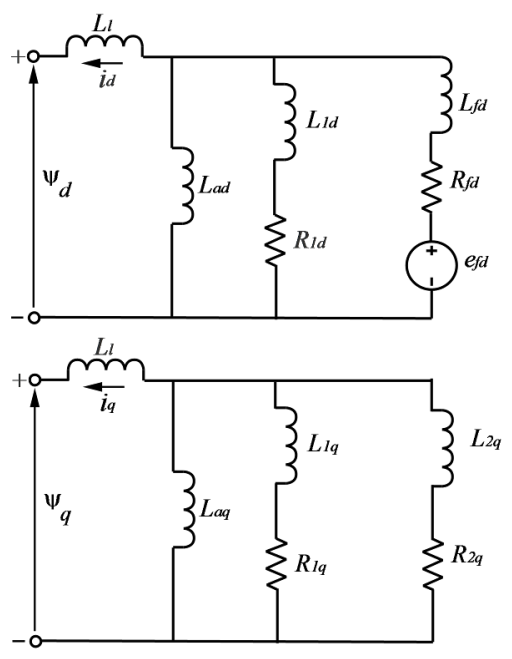

Figure 3. Standard d-and q-axis equivalent circuit.

erational inductance and $L_{q}(s)$ is the q-axis operational inductance, $i_{d}$ and $i_{q}$ are $d$ and $q$ axis currents, $v_{f}$ is the fieldwinding voltage.

The synchronous machine is supposed to be modelled with one damper winding for the $\mathrm{d}$-axis and two dampers windings for the $q$-axis $(2 \times 2$ model $)$. Generally, this model structure considered adequate for stability studies. Fig.3 represents the Standard $d-a n d q-$ axis circuit models. The expressions of the $d$-axis operational parameters are given as follow:

$$
\begin{gathered}
L_{d}(s)=L_{d} \frac{\left(1+s T_{d}^{\prime}\right)\left(1+s T_{d}^{\prime \prime}\right)}{\left(1+s T_{d o}^{\prime}\right)\left(1+s T_{d o}^{\prime \prime}\right)} \\
G_{d}(s)=G_{0} \frac{\left(1+s T_{k d}\right)}{\left(1+s T_{d o}^{\prime}\right)\left(1+s T_{d o}^{\prime \prime}\right)}, \quad G_{0}=\frac{M_{f}}{R_{f}}
\end{gathered}
$$

The expression of the $q$ - axis operational parameters is given as follow:

$$
L_{q}(\mathrm{~s})=L_{q} \frac{\left(1+s T_{q}^{\prime}\right)\left(1+s T_{q}^{\prime \prime}\right)}{\left(1+s T_{q o}\right)\left(1+s T_{q o}\right)}
$$

In the case of silent pole machine, only one $q$-axis rotor circuit (denoted by the subscript 1q) is applicable. The other circuit (denoted by the subscript $2 q$ ) is ignored [14]. Hence, the expression for the $q$-axis operational inductance is:

$$
L_{q}(\mathrm{~s})=L_{q} \frac{\left(1+s T_{q}^{\prime \prime}\right)}{\left(1+s T_{q o}\right)}
$$

Remark1: The main parameters used in Eqs.5-8 are listed in Table. 1

\section{Standstill frequency response test}

The Stand-Still Frequency Response (SSFR) test is developed to study the frequency characteristics of rotating machines at standstill. In the last years, the SSFR method has 
Table 1. Parmeters defintion.

\begin{tabular}{ll}
\hline Parameters & Defintion \\
\hline$L_{d}, L_{q}$ & d-and q- axis synchronous inductance, respectively \\
$T_{d}^{\prime}, T_{d o}^{\prime}$ & d-axis transient open circuit and short-circuit time constant \\
$T_{d}^{\prime \prime}, T^{\prime \prime}{ }_{d o}$ & d-axis subtransient open circuit and short-circuit time constant \\
$T_{q}^{\prime}, T_{q o}^{\prime}$ & q-axis transient open circuit and short-circuit time \\
$T_{q}^{\prime \prime}, T_{q o}^{\prime \prime}$ & q-axis subtransient open circuit and short-circuit time constant \\
$R_{f}$ & field resistances \\
$T_{k d}$ & damper winding leakage time constant \\
\hline
\end{tabular}

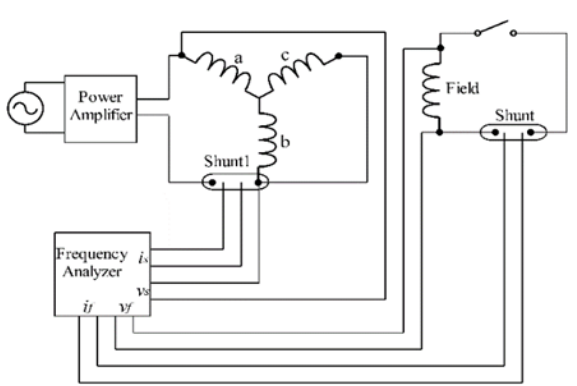

Figure 4. Block diagram of the test frequency (SSFR) Norm IEEE.

been standardized among the main methods for identifying synchronous machines dynamic. SSFR test consists of deducing the characteristics of the operational quantities and depending on frequency [15].

Since in the SSFR method, the tests are performed independently for direct and quadrature axes, it is necessary to align the rotor to two particular positions with respect to the stator. Fig. 4 shows the experimental procedure of the SSFR test according to the recommendation given by the IEEE-Std115 [16].

\section{PSO-based parameters identification}

The basic idea of the parameters identification using PSO algorithms is to convert the problem of parameters estimation into an optimization problem. The unknown parameters are usually set as some particles and a performance function measuring how well the model response fits the system response is built to optimize. The fitness function is defined as:

$$
\text { fitness }=\frac{1}{N} \sum_{i=1}^{N}\left(\mathrm{y}_{i}-\hat{y}_{i}\right)^{2}
$$

$N$ is the the length of the measured outputs vector, $y$ and $\hat{y}$ are the vectors of system and model outputs, respectively. As the $L_{d}(s), L_{q}(s)$ and $G(s)$ are complex quantities, the equation 9 become as:

$$
\text { fitness }=\frac{1}{N} \sum_{i=1}^{N}\left(\mathrm{y}_{\mathrm{Re}}-\hat{y}_{\mathrm{Re}}\right)_{i}^{2}+\frac{1}{N} \sum_{i=1}^{N}\left(\mathrm{y}_{\text {Imag }}-\hat{y}_{\mathrm{Imag}}\right)_{i}^{2}
$$

where, $(.)_{R e}$ and (.) Imag are the real and imaginary parts of the output (.).

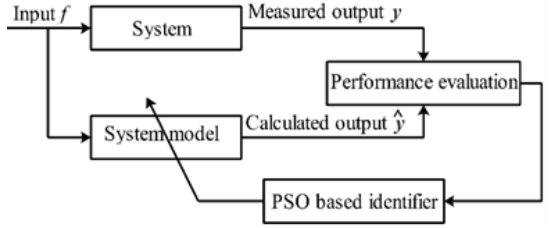

Figure 5. Block diagram of the PSO-based identification approach.

Fig.5 represents the block diagram of the PSO based parameter identification approach. The proposed identification method is described with the following steps:

- Step 1: Measured the response of the real system under an input with variable frequency.

- Step 2: Simulate the system model with initial parameters, under the same inputs as the real system.

- Step 3: Calculates the fitness function of each particle (candidate solutions)

- Step 4: update candidate solutions according to the updating rules in Eqs.1,2.

- Step 5: if the number of iteration is achieved or the convergence criterion is satisfied then stop the optimization, else go to step 2 .

\section{Simulation results}

Numerical tests have been performed to evaluate the performances of the presented method to identify the synchronous machine parameters. Two performance criterions are considered:

- The simple criterion $J_{q}=J_{L q}$ was used to identify the q-axis parameters.

- The mixed criterion $J_{d}=J_{L d}+J_{G}$ was used to identify the d-axis parameters.

Fig.6 shows a comparison between identified and real $q$ - axis transfer functions frequency response using the simple criterion $J_{q}$. the fitness function evolution is depicted in Fig.7. It is clear that the identified parameters are close to their real values. The fitness function decreases rapidly and achieves the convergence criterion after 25 iterations. The method performances with simple criterion are summarized in Table2, which highlight that the identified parameters are close to those given by the constrictor. 


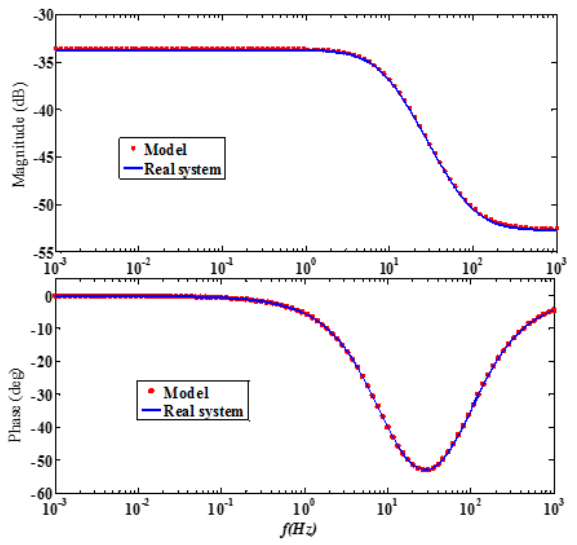

Figure 6. Bode plot of operational inductance Lq(s).

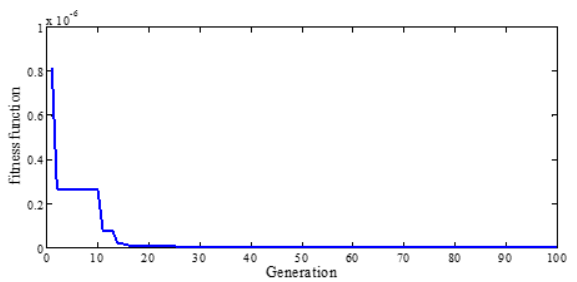

Figure 7. JLq Fitness function evolution.

Table 2. Results of the identification method for Lq(s).

\begin{tabular}{llll}
\hline Parameters & Constrictor & Identified & error \\
\hline$L_{q}$ & 0.0023494 & 0.00234940 & $0.0 \%$ \\
$T_{q}^{\prime \prime}$ & 0.011882 & 0.011880 & $0.016 \%$ \\
$T_{q o}^{\prime \prime}$ & 0.10612 & 0.106119 & $0.00094 \%$ \\
\hline
\end{tabular}

Table 3. Results of the identification method for $\mathrm{Ld}(\mathrm{s})$ and G(s).

\begin{tabular}{llll}
\hline Parameters & Constrictor & Identified & error \\
\hline$G_{0}$ & 2.049 & 2.0490 & $0.0 \%$ \\
$T_{k} d$ & 0.014 & 0.01399 & $0.071 \%$ \\
$L_{d}$ & 0.0048 & 0.00480 & $0 \%$ \\
$T_{d}^{\prime}$ & 0.1 & 0.10005 & $0.05 \%$ \\
$T_{d}^{\prime \prime}$ & 0.01 & 0.009993 & $0.07 \%$ \\
$T_{d o}^{\prime}$ & 1.95 & 1.9500 & $0 \%$ \\
$T_{d o}^{\prime \prime}$ & 0.019 & 0.01899 & $0.05 \%$ \\
\hline
\end{tabular}

Using the mixed criterion $J_{d}$, a comparison between identified and real $d$-axis outputs is given in Figs.8,9. The obtained results clarify that the magnitude and the phase of the identified model are much close to those of the real system, which demonstrates the effectiveness of the-PSO based model parameter identification approach. As shown in Fig.10, the fitness function decreases and achieves the convergence criterion after 140 iterations rather than 25 iterations in the first case. This is due to the high number of parameters to be identified in the second case (seven parameters compared to three in the first case). Table. 3 lists the method performances with mixed criterion. The obtained results confirm the capabilities of the PSO-based algorithm.

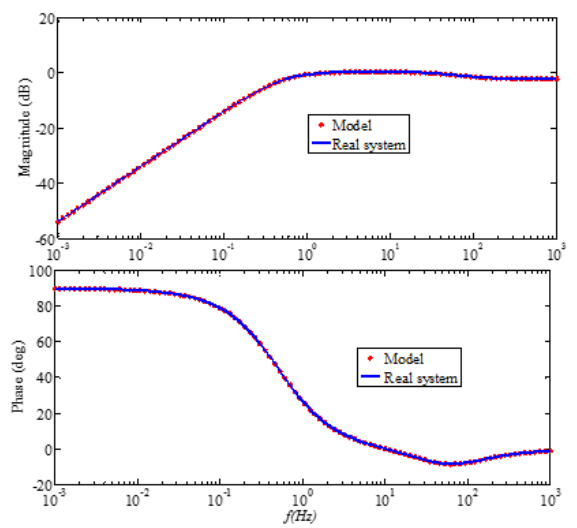

Figure 8. Bode plot of operational inductance G(s).

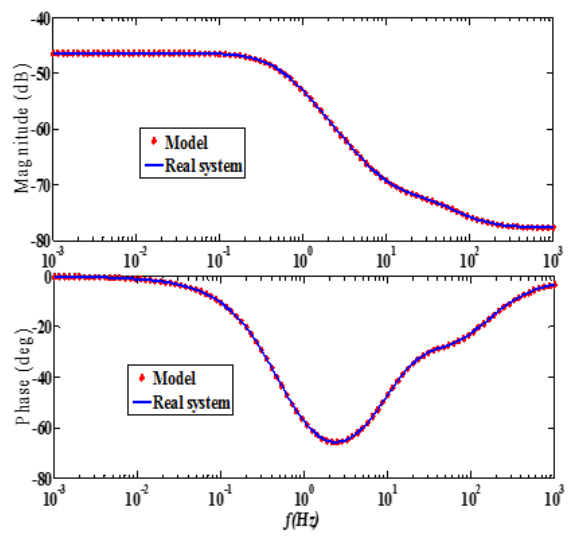

Figure 9. Bode plot of operational inductance $\operatorname{Ld}(\mathrm{s})$.

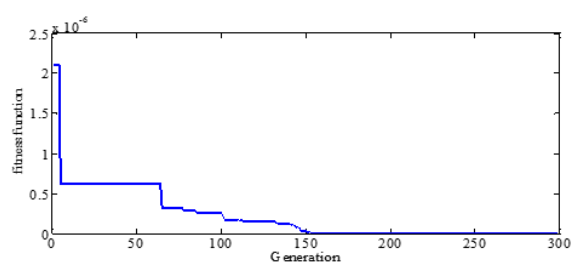

Figure 10. JLd+JG Fitness function evolution

\section{Conclusion}

In this paper, an approach based on particle swarm optimization algorithm is presented for synchronous machine operational parameters identification using the Standstill Frequency Response test simulation data. The PSO algorithm is applied to minimize the quadratic criterion. Simulation results are provided to validate the effectiveness of this identification method. The obtained results show that PSO algorithms are capable of finding the main parameters of the synchronous machine. It is shown also that al- 
though convergence time is adversely effected by the number of parametrs to be identified, the algorithm keeps its efficiency.

\section{References}

[1] M. Hasni, O. Touhami, R. Ibtiouen, M. Fadel, S. Caux, Mathematics and Computers in Simulation 81, 277 (2010)

[2] J. Melgoza, G. Heydt, A. Keyhani, B. Agrawal, D. Selin, IEEE Transactions on Energy Conversion 16, 49 (2001)

[3] R. Fard, M. Karrari, O. Malik, IEEE Transactions on Energy Conversion 20, 852 (2005)

[4] A. Belqorchi, U. Karaagac, J. Mahseredjian, I. Kamwa, IEEE Transactions on Power Systems 34, 2261 (2019)

[5] H.B. Karayaka, A. Keyhani, G. Heydt, B. Agrawal, D. Selin, IEEE Transactions on Energy Conversion 16, 305 (2001)

[6] M.E. Jahromi, M. Rasouli, M. Ranjbar, Synchronous generator parameters estimation using genetic algorithm via DC decay field tests, in 2005 IEEE Russia Power Tech (IEEE, 2005), https : //doi .org/10. 1109\%2Fptc. 2005.4524728

[7] T. Niewierowicz, R. Escarela-Perez, E. CamperoLittlewood, Computers \& Electrical Engineering 29, 505 (2003)

[8] P. Kou, J. Zhou, C. Wang, H. Xiao, H. Zhang, C. Li, Engineering Applications of Artificial Intelligence 24, 1227 (2011)
[9] L. Liu, W. Liu, D.A. Cartes, Engineering Applications of Artificial Intelligence 21, 1092 (2008)

[10] G. Hutchison, K. Harmer, D. Giaouris, B. Zahawi, B. Stedall, Synchronous machine parameter identification using particle swarm optimization, in 5th IET International Conference on Power Electronics, Machines and Drives (PEMD 2010) (Institution of Engineering and Technology, 2010), https://doi . org/10.1049\%2Fcp . 2010.0061

[11] I. Pan, S. Das, ISA Transactions 62, 19 (2016)

[12] A. Lari, A. Khosravi, F. Rajabi, ISA Transactions 53, 517 (2014)

[13] M.P. Aghababa, Soft Computing 20, 4055 (2015)

[14] P. Kundur, in Power System Stability and Control (CRC Press, 2007), https://doi .org/10.1201\% 2F9781420009248.sec2

[15] H. Radjeai, A. Barakat, S. Tnani, G. Champenois, Identification of synchronous machine by Standstill Frequency Response (SSFR) method - influence of the stator resistance -, in The XIX International Conference on Electrical Machines - ICEM 2010 (IEEE, 2010), https : //doi .org/10 . 1109\% 2Ficelmach. 2010.5608146

[16] IEEE standard procedures for obtaining synchronous machine parameters by standstill frequency response testing (supplement to ANSI/IEEE std 115-1983, IEEE guide: Test procedures for synchronous machines), https : //doi . org/10.1109\% 2Fieeestd.1987.79637 